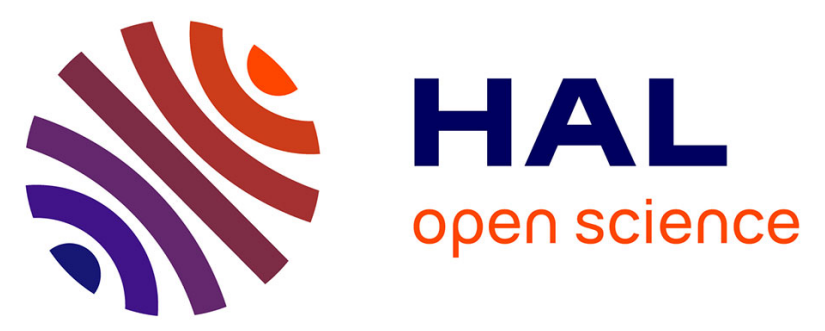

\title{
MRI contrast variation of thermosensitive magnetoliposomes triggered by focused ultrasound: a tool for image-guided local drug delivery
}

Cyril Lorenzato, Alexandru Cernicanu, Marie-Edith Meyre, Matthieu Germain, Agnès Pottier, Laurent Levy, Baudouin Denis de Senneville, Clemens Bos, Chrit Moonen, Pierre Smirnov

\section{To cite this version:}

Cyril Lorenzato, Alexandru Cernicanu, Marie-Edith Meyre, Matthieu Germain, Agnès Pottier, et al.. MRI contrast variation of thermosensitive magnetoliposomes triggered by focused ultrasound: a tool for image-guided local drug delivery. Contrast Media and Molecular Imaging, 2013, 8 (2), pp.185-192. hal-01578206

\author{
HAL Id: hal-01578206 \\ https://hal.science/hal-01578206
}

Submitted on 28 Aug 2017

HAL is a multi-disciplinary open access archive for the deposit and dissemination of scientific research documents, whether they are published or not. The documents may come from teaching and research institutions in France or abroad, or from public or private research centers.
L'archive ouverte pluridisciplinaire HAL, est destinée au dépôt et à la diffusion de documents scientifiques de niveau recherche, publiés ou non, émanant des établissements d'enseignement et de recherche français ou étrangers, des laboratoires publics ou privés. 
MRI contrast variation of thermosensitive magnetoliposomes triggered by focused ultrasound: a tool for image guided local drug delivery.

Cyril Lorenzato $^{1,2}$, Alexandru Cernicanu ${ }^{3}$, Marie-Edith Meyre ${ }^{4}$, Matthieu Germain ${ }^{4}$, Agnès Pottier ${ }^{4}$, Laurent Levy ${ }^{4}$, Baudouin Denis de Senneville ${ }^{1,2},{\text { Clemens } \text { Bos }^{2}, \text { Chrit Moonen }^{1,2} \text {, Pierre Smirnov }}^{1,2}$

${ }^{1}$ Laboratory for Molecular and Functional Imaging: from Physiology to Therapy, FRE 3313 CNRS / University Bordeaux Segalen, France

${ }^{2}$ Image Sciences Institute, University Medical Center Utrecht, Heidelberglaan 100, 3584 CX Utrecht,

${ }^{3}$ Philips Healthcare, 33 rue de Verdun, 92150 Suresnes, France.

${ }^{4}$ Nanobiotix, 60 rue de Wattignies, bâtiment B, 3 ème étage, 75012 Paris, France.

Correspondence should be addressed to Pierre Smirnov

E-mail: pierre_smirnov@hotmail.com

Tel: $\quad+31887550922$

Fax: $\quad+31302539032$

Short title: MRI drug delivery evaluation with thermosensitive magnetoliposomes

Keywords: MRI; Local drug delivery; Ultrasound; Thermosensitive liposomes; 


\begin{abstract}
Improved drug delivery control during chemotherapy is a major concern to increase their therapeutic index. Drug accumulation in solid tumor can be visualized using MRI contrast agent such as iron oxide nanoparticles encapsulated in liposomes. Once accumulated in tumor, the combination of a thermosensitive composition with an external source of activation allows local release of drug. MRI guided-High intensity focused ultrasound (HIFU) represents a non invasive technique to generate local hyperthermia for drug release of thermosensitive magnetoliposomes (TSM).
\end{abstract}

In this study we performed encapsulation of ultrasmall superparamagnetic iron oxide nanoparticles (USPIO) in thermosensitive liposomes to obtain TSM. Magnetic behavior of this MRI contrast agent was observed during TSM membrane permeabilization. For this, measurement of transverse and longitudinal relaxivities on MRI, and real time experiments were performed on TSM samples loaded with USPIO during heating using a water bath or HIFU. Results showed significant differences for MRI signal enhancement and relaxivities ratios before and after heating, which were absent for non-thermosensitive liposomes and free nanoparticles used as controls. Thus, incorporation of USPIO as MRI-contrast agents into thermosensitive liposomes should, besides TSM tumor accumulation, allows the visualization of TSM membrane permeabilization upon temperature elevation. In conclusion, HIFU under MR image guidance in combination with USPIO loaded thermosensitive liposomes as drug delivery system has the potential for a better control of drug delivery and to increase the therapeutic index of chemotherapy. 


\section{INTRODUCTION}

Chemotherapy for tumor treatment is based on systemic administration of drugs which reach blood stream and are distributed in the whole body. Biodistribution of these free drugs is a main concern as it may results in a narrowed therapeutic index of these types of treatment. Indeed, chemotherapeutic agents target all rapid dividing cells and are not specific for cancer cells leading to harm healthy tissues but also presenting specific interactions with biological molecules leading to an enhanced toxicity towards specific organs (e.g. cardiotoxicity for doxorubicin (1)). Currently, the majority of deaths due to cancer are the result of solid tumors. Indeed, for most advanced cancers, chemotherapy remains the standard treatment, but is rarely completely curative for solid tumors (2). It is therefore necessary to improve the specificity of existing treatments by targeting the drug to concentrate preferentially into the tumor (macroscopic targeting), and reach targeted cancer.

A field on the crossroads of nanotechnology and biotechnology is the encapsulation of drugs in nanocarriers (3-5). Doing so results in modifying its biodistribution to optimize delivery of the appropriate concentration of drug to the appropriate site. Accumulation of the drug-loaded nanocarriers at the tumor site is favored by the enhanced permeability and retention (EPR) effect (6). This EPR effect is a property favorising extravasation of large object in the tumor due to its abnormal vascularization with larger fenestration of vascular endothelium and lack of lymphatic drainage. Encapsulation provides also a protection against drug metabolisation and inactivation in plasma.

Among the different nanocarriers available, liposomes are still of great interest. Discovered by Alec Bangham in the beginning of the $1960^{\text {th }}(7)$, many studies have been made of the use of liposomes as drug carrier $(8,9)$. Their structure made of a phospholipid bilayer surrounding an aqueous compartment is biocompatible and allows the encapsulation of hydrophilic or hydrophobic drugs in a tunable system (size, membrane composition, steric coating avoid captation by 
reticuloendothelial system, targeting agent addition.). Due to these advantages liposome formulations are increasingly considered for treatment against cancer and used clinically $(10,11)$.

In order to improve drug bioavailability once accumulated in the tumor, nanocarriers can be modified to be sensitive to an external and non-invasive stimulus. In this way, phospholipid composition of liposomes membrane can be adjusted to be temperature sensitive (12). Indeed, a phase transition occurs for phospholipids between a rigid, gel-like structure and a fluid, permeable liquid-crystalline phase, at a specific temperature, the melting temperature $T_{m}$. Generally, formulations of thermosensitive liposomes are mainly composed of phosphatidylcholines presenting a phase transition temperature $\mathrm{T}_{\mathrm{m}}$ in the range of mild hyperthermia $\left(41^{\circ} \mathrm{C}-43^{\circ} \mathrm{C}\right)$. The thermosensitive liposomes obtained are able to locally produce a burst release of their payload under activation. A promising approach to control drug release is to combine thermosensitive liposomes with high intensity focused ultrasound (HIFU). The energy of mechanical vibration of ultrasound waves is converted in heat in the targeted tissue and was originally designed for thermal ablation (13). Using this technology to apply a local and moderate temperature elevation (141515), visualizable by MRI allows thermosensitive liposomes permeabilization and drug release without toxicity for surrounding tissues (16). Such mild energy ultrasound, even if non toxic for cell, create a transient permeabilization of their membrane which could favor drug cellular uptake once released from thermosensitive liposomes.

A major point for the design of local activation of drug delivery system (DDS) is to ensure a release of the encapsulated drug at the right time, at the right place. To ensure such a level of control, a monitoring of DDS accumulation in tumor and of its permeabilization under activation are required. Several approaches are explored to monitor nanocarrier accumulation in tumor and to follow the release of their content under external activation. Use of MRI contrast agents is a method of choice for HIFU which is already coupled to MRI for temperature mapping. 
Paramagnetic contrast agents were first used to follow the release of liposome contents, e.g. by means of changes of the water access to chelated gadolinium (17). Several studies were done in order to improve their properties $(12,18)$ (sensitivity and/or stability) and confirmed that, in principle, paramagnetic contrast agents such as gadolinium are suitable to follow a drug delivery process. They are not appropriate, however, to follow the macro-biodistribution as their abilities to create a contrast are mostly counteracted by the liposome's bilayer before they are released.

Superparamagnetic contrast agents such as Ultra Small Iron Oxide Particles (USPIO) should be detectable before release occurs, because of their influence on magnetic field homogeneity. USPIO have been encapsulated in liposome to form so called magnetoliposomes. Accumulation of these nanocarriers in tissue is easily monitored by MRI due to their ability to considerably reduce transversal relaxation time. The $\mathrm{T}_{2}{ }^{*}$ relaxivity effect is stronger for clustered than for dispersed USPIO particles, suggesting that a detectable MR-signal change should be present upon permeabilization of liposomes. In contrast, clustering reduces longitudinal relaxivities of iron oxide nanoparticles, as was demonstrated for cellular internalization (19-21) or drug carriers such as liposomes. Hence, a system of thermosensitive magnetoliposomes (TSM) encapsulating anticancer drugs and nanoparticles $(22,23)$, can be an attractive candidate for image guided drug delivery.

The principal aim of this article was to demonstrate the feasibility of the monitoring of thermosensitive magnetoliposomes permeabilization by MRI during activation by HIFU. For this, we hypothesized that TSM permeabilization leads to a modification of agglomeration state of USPIO generating an increase of $\mathrm{T}_{2} *$ relaxation time and also an improved detectability of $\mathrm{T}_{1}$-weighted signal enhancement. MRI signal enhancement, relaxation times and relaxivities of TSM samples, before, during and after heating were measured, first in a water bath to established proof of concept and finally with HIFU heating into a gel for dynamic follow up. The system presented is a step towards monitoring both phases of drug delivery: accumulation and release in the targeted tissue. 


\section{RESULTS AND DISCUSSION}

\section{MR signal modifications after water-bath heating}

The iron oxide-containing thermosensitive liposomes were first characterized by cryo-transmission electron microscopy (cryo-TEM) (figure 1a,b). Since iron oxide nanoparticles are electron-dense particles, aggregation state of the USPIOs could be observed without staining. After synthesis, the iron oxide nanoparticles were encapsulated in liposomes of 100-200 nm (figure 1a) as clusters of nanoparticles. Detailed analysis of TSM size distribution, measured by Dynamic Light Scattering (figure 1c), showed a mean hydrodynamic diameter of $203 \mathrm{~nm}$ with a polydispersity index of 0.14 . Before heating (figure 1a), USPIO were aggregated into clusters with a diameter slightly larger than $50 \mathrm{~nm}$. After heating, nanoparticles could be identified as single particles, dot-like with a diameter smaller than $10 \mathrm{~nm}$ (figure 1b, empty arrow). Because of the diffusion limit imposed by the liposome membrane, these two extreme sizes give us the range $(10$ to $50 \mathrm{~nm})$ for the iron oxide nanoparticle agglomerate size change observable during MRI experiments. The phospholipids membranes were discernible before heating and showed smooth and round contours black arrow after the heating experiment, lipid membranes exhibited polyhedral structures demonstrating that a phase transition occurred during the heating process.

Typical $\mathrm{T}_{1}$-weighted images obtained before and after the water bath heating show a large MRI signal enhancement of $83 \pm 4 \%$ for TSM, figure 3 . This was the case for neither USPIO nor Non-thermosensitive liposomes (NTSM) where enhancements of $-7 \pm 4 \%$ and $-1 \pm 3 \%$ were observed respectively. $\mathrm{T}_{1}$-weighted gradient echo imaging is well adapted for fast image acquisition. Hence, this sequence can be used in order to monitor the $T_{1}$ relaxation change during heating, to detect the liposome membrane permeabilization and provide an indication for release of a co-encapsulated anticancer drug. To characterize the effect of iron oxide nanoparticles-containing thermosensitive liposomes on the MR signal, relaxivities were measured, before and after heating, 
figure 2. In figures 2 (a-c), respectively $1 / \mathrm{T}_{1}, 1 / \mathrm{T}_{2}$ and $1 / \mathrm{T}_{2} *$ were plotted function of the concentration in $\mathrm{Fe}(\mathrm{III})$ of the samples, before and after heating. Longitudinal relaxivity of TSM increased from $\mathrm{r}_{1}=1.6 \pm 0.3$ to $\mathrm{r}_{1}=4.6 \pm 0.7 \mathrm{mM}^{-1} . \mathrm{s}^{-1}$ (figure $2 \mathrm{~d}$ ). At the same time, transversal relaxivities $r_{2}$ and $r_{2^{*}}$ decreased significantly from $r_{2}=221.3 \pm 15.3$ to $r_{2}=139.1 \pm 4.6 \mathrm{mM}^{-1}$. $\mathrm{s}^{-1}$ (figure 2e) and from $\mathrm{r}_{2^{*}}=248.8 \pm 15.9$ to $\mathrm{r}_{2^{*}}=152.5 \pm 13.4 \mathrm{mM}^{-1} \cdot \mathrm{s}^{-1}$ (figure $2 \mathrm{f}$ ). While TSM samples still exhibit significant differences after the cooling period back at room temperature, free USPIO relaxivities remain stable: $r_{1}=15.7 \pm 5.3$ and $r_{1}=13.5 \pm 2.4 \mathrm{mM}^{-1} \cdot \mathrm{s}^{-1}, \mathrm{r}_{2}=195.0 \pm 3.3$ and $\mathrm{r}_{2}=185.6 \pm 6.5 \mathrm{mM}^{-1} \cdot \mathrm{s}^{-1}$, and $\mathrm{r}_{2^{*}}=209.8 \pm 14.3$ and $\mathrm{r}_{2^{*}}=189.0 \pm 13.4 \mathrm{mM}^{-1} \cdot \mathrm{s}^{-1}$. Values are reported before and after heating, respectively. As the ratio $r_{2^{*}} / r_{1}$ is a measure of the efficiency of an MRI contrast agent (24) we looked at this value before and after heating for TSM and free USPIO. For TSM the $r_{2^{*}} / r_{1(\mathrm{TSM})}=164.7$ decreased to $r_{2 *} / r_{1(\mathrm{TSM})}=33.5$, while for free USPIO it remained unchanged $\left(\mathrm{r}_{2 *} / \mathrm{r}_{1(\mathrm{USPIO})}=14.5\right.$ before heating to $\mathrm{r}_{2 *} / \mathrm{r}_{1 \text { (USPIO })}=14.6$ after heating) . Signal noise ratio (SNR) of MRI sequences used for $\mathrm{T}_{1}$ measurement was equal to $65.5 \pm 1.8$ for the longest inversion time and equal to $9.7 \pm 0.9$ and $8.2 \pm 0.9$ for $\mathrm{T}_{2}$ and $\mathrm{T}_{2}{ }^{*}$ measurement for the longest echo time.

The high value of $\mathrm{r} 2 * / \mathrm{r} 1$ observed for USPIO encapsulated in liposomes before heating is due to a high $\mathrm{r}_{2} *$ value $\left(\mathrm{r}_{2}{ }^{*}=248.8 \mathrm{mM}^{-1} \cdot \mathrm{s}^{-1}\right)$ and mainly to a low $\mathrm{r}_{1}$ value, as it can be noted that before heating, nanoparticles clustered into liposomes showed a longitudinal relaxivity 10 times lower than free nanoparticles. The limited $r_{1}$ effect of USPIO internalized in liposomes observed in this study could be explained by water access, with two different pools of protons: a small pool confined into liposomes with high $r_{1}$, due to the internalized contrast agent, and a large external pool with low $\mathrm{r}_{1}$ (20). If the residence time, $\tau_{\mathrm{D}}$, of protons in the liposomes becomes larger than its relaxation time, the relaxation effect of the iron oxide nanoparticles confined in the liposomes saturates. Because the phospholipidic layer surrounding the aggregated USPIO limits diffusion, exchange of water protons between both pools is restricted. Thus the longitudinal relaxivity of the particles in liposomes is reduced, and the apparent $r_{1}$ relaxivity of iron oxides in liposomes is lower compared with freely 
dispersed contrast agents. After heating in a water bath at $45^{\circ} \mathrm{C}$, the phospholipid phase transition, occurring at $43^{\circ} \mathrm{C}$ leads to the permeabilization of the lipid barrier, and allows external water access. It can be hypothesized that upon permeabilization, a change of the internal aqueous phase may modify the internal ionic strength of liposomes upon entry of external buffer, thus modifying the agglomeration state of negatively charged iron oxide nanoparticles in the liposome core and leading to the decrease of $\mathrm{r} 2 *$.

In this experiment we used the observation that for $\left(\mathrm{T}_{2}{ }^{*}\right)$ proton dephasing in the presence of magnetic particles, two major regimes can be described, depending on the size of the particle (25): the so-called Motional Averaging Regime (MAR) and the Static Dephasing Regime (SDR). In the motional averaging regime, that applies to small particles (diameter between 5 to $20 \mathrm{~nm}$ ), a proton experiences, because of Brownian motion, the field disturbance of different particles. Hence, local field effects are averaged out leading to a relatively long $\mathrm{T}_{2}{ }^{*}$ (26). For larger particles, or nanoparticle clusters, at identical iron concentration as in our experiment, the distance between the particles increases, so limiting the averaging effect due to diffusion, and decreasing $\mathrm{T}_{2}{ }^{*}$. The proton experiences the field disturbance of one particle as if it were stationary, i.e. the so called SDR. Particles with a radius in the range of 5 to $25 \mathrm{~nm}$, as observed using TEM, theoretically correspond to the MAR region. The clustered particles on the other hand should lead to $\mathrm{T}_{2}{ }^{*}$ changes closer to that corresponding to the SDR. In addition, $\mathrm{T}_{2}$ and $\mathrm{T}_{2}{ }^{*}$ values observed for the TSM are very close, indicating that the MAR applies $(27,28)$

The phospholipid membrane played also an important role in the $\mathrm{T}_{2}{ }^{*}$ evolution. Chantal Paquet et al. (29) described the effect of the encapsulation of magnetic particles with a hydrophilic coating holding protons at the surface of a particle for a longer period of time. Similar to these hydrophilic coatings, the liposomes membrane encapsulates the water around the particles limits water diffusion locally. Hence the $\mathrm{T}_{2}{ }^{*}$ values of liposomes containing USPIO, such as TSM which have a diameter 
of about around $200 \mathrm{~nm}$ (figure 3c) would tend towards the SDR, further increasing the $\mathrm{T}_{2}{ }^{*}$ contrast change after release.

\section{Dynamic MRI}

A dynamic study was done in order to demonstrate the possibility to follow the liposome membrane permeabilization over time. After drift correction, temperature on MR thermometry was well correlated with the temperature measured by the optical fiber. However, over the extent of the phantom, a mean shift of $1.89 \pm 0.6{ }^{\circ} \mathrm{C}$ was present with respect to the probe in the center. This was most likely due to inhomogeneous heating of the phantom gel, from the outside. The shift was substantially smaller if regions near the optical probe were considered.

The magnitude of the MR-signal of the dynamic series is $\mathrm{T}_{1}$-weighted $(\mathrm{TR}=300 \mathrm{~ms}, \mathrm{TE}=10 \mathrm{~ms})$ and so will reflect the $\mathrm{T}_{1}$ changes over time, figure $4 \mathrm{a}$. For free USPIO the magnitude signal follows the temperature evolution, figure $4 \mathrm{~b}$, because as temperature rises the interaction between water and nanoparticles increases. The signal behavior from the TSM seemed to have the same shape except during the period corresponding to the phase transition temperature where a positive shift of $50 \%$ occurred. Here, the $T_{1}$ relaxation time decreased because of the change of internal aqueous phase upon membrane permeabilization and subsequent change of the agglomeration state of iron oxide nanoparticles.

It can be argued that there is some $\mathrm{T}_{2}{ }^{*}$-weighting in the signal, and that a $\mathrm{T}_{2} *$ increase after release, which was demonstrated above, is responsible for the signal change. However, based on values measured during the experiment $\left(\mathrm{T}_{2} * \geq 14.4 \pm 0.4 \mathrm{~ms}\right.$, for all time points), the influence of $\mathrm{T}_{2} *$ on the signal magnitude enhancement was estimated, and it was demonstrated that for the TSM a clear $\mathrm{T}_{1}$-weighted signal enhancement effect is observed. 
Figure 5a shows $r_{2}{ }^{*}$ variation versus time, each calculated from the $T_{2} *$ values of four samples with different iron concentrations. In water, the evolution of $\mathrm{r}_{2^{*} \text { (USPIO) }}$ was linearly correlated with temperature. For thermosensitive liposomes, however, $\mathrm{r}_{2^{*}(\mathrm{TSM})}$ showed a sigmoidal variation between 42 and $44.5^{\circ} \mathrm{C}$ in addition to the baseline variation with temperature seen for free USPIO. This hysteresis took place in 7 minutes with an inflexion point located near the phase transition temperature $\left(\mathrm{T}_{\mathrm{m}}=43^{\circ} \mathrm{C}\right)$ and had a sigmoidal behavior similar to a classical phase transition from thermosensitive liposome monolayer and, thus, is very likely to correlate with the liposome permeabilization (30).

Alternatively, $\mathrm{T}_{1}$-weighted enhancement and $\mathrm{r}_{2^{*}}$ can be plotted as a function of temperature (figure $4 \mathrm{~b}$ and $5 \mathrm{~b}$ respectively), to better demonstrate hysteresis: during the cooling period, $\mathrm{r}_{2 * \text { (TSM) }}$ did not return to its original value. This suggests that the iron oxide nanoparticles environment change occurring upon heating is irreversible, corresponding to dilution of the internal aqueous phase (entry of buffer upon membrane permeabilization), leading to a change of the agglomeration state of iron oxide nanoparticles.

\section{Proof of concept: local HIFU heating experiment.}

This last experiment was done to demonstrate the feasibility of registering spatially controlled release of USPIO from the nanocarriers by observing $\mathrm{T}_{2} *$ variation, using HIFU for heating. Before heating experiment, the center polyacrylamide gel doped by TSM showed a mean relaxation time $\mathrm{T}_{2}{ }^{*}$ of $6.5 \pm 0.7 \mathrm{~ms}$ (figure $6 \mathrm{~b}$ ).

$\mathrm{T}_{2} *$ increased to $57 \pm 18.4 \mathrm{~ms}$ for a $5 \times 5 \mathrm{~mm}$ ROI that received 4 heating cycles. At a different position, one cycle of heating was applied, which leads to a smaller increase of $\mathrm{T}_{2}{ }^{*}$, viz. $20.9 \pm 6.2$ $\mathrm{ms}$, on a smaller $3 \times 3 \mathrm{~mm}$ ROI (figure $6 \mathrm{~d}$ ). As expected a corresponding positive enhancement could be also observed on the magnitude images (figure 6c), but seemed to be relatively smaller (65.1 \pm $19.5 \%$ in the center ROI). As water could not move freely within the gel, the diffusion was smaller 
than in a liquid sample used in the first step characterization which could explain a reduced change in the relaxation time $T_{1}$. This also applies to $T_{2} *$ value changes because transversal relaxation time is diffusion-dependent as described in the outer-sphere relaxation theory. In this case, a low polyacrylamide concentration was used $(2 \%)$, to limit $\tau_{\mathrm{D}}$ and maintain $\mathrm{T}_{2}{ }^{*}$ variation effect. The $\mathrm{T}_{1}$ and $\mathrm{T}_{2} *$ variation occurring in-vivo during the carrier permeabilization, still remains to be studied, as the diffusion coefficient and or the electrostatic environment may differ.

Finally, we demonstrated that we can spatially control and visualize the membrane permeabilization of thermosensitive magnetoliposomes by MRI, showing two distinct regions of $T_{2} *$ change corresponding to different positions of the US transducer.

Thus, as for the $\mathrm{T}_{1}$-weighted imaging mentioned above, the use of $\mathrm{T}_{2}{ }^{*}$ measurement may be an interesting and sensitive method for evaluating the membrane permeabilization of thermosensitive magnetoliposomes and subsequent release of a co-encapsulated anticancer drug from liposomes. 


\section{CONCLUSIONS}

In this study, an approach using thermosensitive magnetoliposomes to monitor local drug delivery by MRI was presented. Relaxation properties of TSM were characterized and we have shown that thermosensitive liposomes containing USPIO nanoparticles had different MR-contrast properties than free USPIOs. The permeabilization of the liposome membrane upon heating at a temperature superior to the phospholipid phase transition temperature leads to a change of the internal buffer of liposomes and to a modification of the iron oxide nanoparticles confinement. A large 5-fold (decrease of the $r_{2} * / r_{1}$ ratio of TSM is then observed upon heating. Hence, we demonstrated that, when encapsulated in liposomes, USPIO nanoparticles are efficient contrast agent to follow the lipid membrane permeabilization over time during a heating experiment, by observing the decrease in $T_{1}$ or the increase in $\mathrm{T}_{2} *$ relaxation time. We showed that membrane permeabilization of TSM and subsequent drug release could be locally triggered and observed by combining HIFU heating and dynamic MRI. Moreover, incorporation of iron oxides as MRI-contrast agents into the thermosensitive liposomes system not only offers the possibility to visualize the drug delivery but also to track drug carriers before inducing the release process. In conclusion, this approach using TSL including superparamagnetic contrast agents can be used for MR-guided HIFU mediated local drug delivery of small molecular anticancer drugs. 


\section{EXPERIMENTAL}

\section{Synthesis of thermosensitive liposomes containing iron oxide nanoparticles.}

Iron oxide superparamagnetic nanoparticles, thermosensitive and non-thermosensitive liposomes loaded with nanoparticles were provided by Nanobiotix Laboratory (Paris, France). Briefly, $5 \mathrm{~nm}$-sized nanoparticles are synthesized by coprecipitation of ferrous and ferric ions (31). Nanoparticles are coated with a chemical envelope bringing a negative surface charge at $\mathrm{pH} 7$ to ensure stability at neutral $\mathrm{pH}$. Thermosensitive liposomes encapsulating Ultra Small superparamagnetic Iron Oxide nanoparticles (USPIO) were prepared using the lipid film re-hydration method (7, 32). Briefly, dipalmitoylphosphatidylcholine (DPPC), hydrogenated soybean phosphatidylcholine (HSPC), cholesterol (Chol) and distearylphosphatidylethanolamine-[methoxy(polyethylene glycol)-2000] (DSPE-PEG2000) are solubilized in chloroform in the molar ratio 100:33:27:7 (DPPC:HSPC:Chol:DSPE-PEG). The solvent is then evaporated under a nitrogen flow. Hydration of the lipid film with a USPIO solution is performed at $55^{\circ} \mathrm{C}$, then 10 freeze-thaw cycles are applied (by plunging the sample in liquid nitrogen, then thawing at $55^{\circ} \mathrm{C}$ in a water bath) and finally the liposomes solution is extruded 5 times on a $0.45 \mu \mathrm{m}$ poly(vinylidene fluoride) membrane and 10 times on a $0.22 \mu \mathrm{m}$ poly(ether sulfone) membrane filter. Non-encapsulated iron oxide nanoparticles are eliminated by Size Exclusion Chromatography on a 15cm-height column filled with Sephacryl S1000 gel (eluant: HEPES 25mM $\mathrm{pH}$ 7.4). The phase transition temperature of the as-prepared iron oxide-loaded liposomes was $\mathrm{T}_{\mathrm{m}}=43^{\circ} \mathrm{C}$, measured by Dynamic Light Scattering (Zetasizer Nano ZS, Malvern) as a function of temperature. Hydrodynamic diameter of liposomes was $203 \mathrm{~nm}$ with a polydispersity index of 0.14 , as measured by Dynamic Light Scattering.

\section{Gels \& samples preparations}


The different concentrations used of liposomes or nanoparticles solution were prepared by serial dilution. Thermosensitive, non-thermosensitive liposomes and nanoparticles (TSM, NTSM and Np respectively) were diluted in $1.5 \mathrm{ml}$ Eppendorf tubes $\left(\left[\gamma-\mathrm{Fe}_{2} \mathrm{O}_{3}\right]=5,10,20\right.$ and $40 \mu \mathrm{g} \cdot \mathrm{ml}^{-1}$ or $[\mathrm{Fe}]$ $0.06,0.13,0.25,0.5 \mathrm{mM}$, respectively) with deionized water containing $25 \mathrm{mM}$ of HEPES. For the experiment observing relaxivity changes before and after heating, the tubes were included in 4\%-agarose gel for stabilization and MR field homogeneity. For the dynamic MRI experiments, the same samples were prepared and tubes were mounted directly in a Bioblock Scientific MRI compatible water bath.

HIFU experiments were done using a polyacrylamide gels $(2 \%)$ with $4 \%$ of precipitated silica containing $[\gamma-\mathrm{Fe} 2 \mathrm{O} 3]=20 \mu \mathrm{g} / \mathrm{ml}$ from TSM. Five milliliter of this gel was included in bigger agarose gel (4\%-2\% silicone dioxide) of $100 \mathrm{ml}$. Acrylamide, ammonium persulfate, N,N,N',N '-Tetramethylethylenediamine (TEMED) and Silicone dioxide were purchased from Sigma-Aldrich ${ }^{\circledR}$ (St. Louis, MO). SELECT Agar powder was purchased from Invitrogen ${ }^{\mathrm{TM}}$ Life Technologies. The ultrasound setup is described below.

\section{MRI and Ultrasound experiments}

MRI experiments were performed on a 1.5 Tesla whole-body clinical magnet (Achieva, Philips Medical Systems, Best, Netherlands). Inversion recovery sequences were used for $\mathrm{T}_{1}$ measurement $(\mathrm{TR} / \mathrm{TI}$ first $/ \Delta \mathrm{TI}=2000 / 50 / 100 \mathrm{~ms}, \quad \mathrm{TE}=10 \mathrm{~ms}, \quad \mathrm{NEX}=2, \quad$ resolution $=1 \times 1 \times 3 \mathrm{~mm}$, matrix $=128 \times 168)$. Transverse relaxation time $T_{2}$ measurement were performed using a multi spin-echo sequence $\quad(8$ echoes, $\quad$ TR $/$ TE first $/ \Delta \mathrm{TE}=300 / 10 / 10 \mathrm{~ms}, \quad \mathrm{NEX}=4$, resolution $=1 \times 1 \times 3 \mathrm{~mm}, \quad$ matrix $=128 \times 168)$. Gradient echo sequences were used for $\mathrm{T}_{2}{ }^{*}$ measurement, with a flip angle $=40^{\circ}, 10$ echoes, TR $/$ TE first $/ \Delta \mathrm{TE}=300 / 10 / 10 \mathrm{~ms}, \mathrm{NEX}=4$, resolution $=1 \times 1 \times 3 \mathrm{~mm}$, matrix $=128 \times 168$ ), yielding 3 slices every $45 \mathrm{~s}$. 
Ultrasound experiments were performed with an in-house-designed, single-channel spherical focused ultrasound transducer (Imasonic SA) integrated in the bed of the MRI. The transducer had a focal length of $80 \mathrm{~mm}$, with operating frequency of $1.5 \mathrm{MHz}$. The focal spot is a revolution ellipsoid of $1 \times 6 \mathrm{~mm}$. HIFU heating was performed with acoustic power of $20 \mathrm{~W}$ during 5 minutes resulting in a temperature stable around $45^{\circ} \mathrm{C}$ during at least 2 minutes. Four HIFU sonications were done, three located in the center of the polyacrylamide gel and one shifted in the upper-right of the polyacrylamide gel. Measurements presented here were done before and after 20 minutes consecutively to the two HIFU sonications.

Proton resonance frequency-based temperature MRI was calculated using an echo time of $14 \mathrm{~ms}$ (near the $\mathrm{T}_{2} *$ values of the observed nanoparticles). Briefly, as the phase variation in gradient-echo images is proportional to the temperature variation, a simple subtraction of each new phase image from a reference phase image provided the temperature evolution on time. Phase Drift due to the slow decrease of the static field during a dynamic MRI experiment was corrected using a ROI placed in a reference gel at constant temperature. In addition, an independent measure of temperature was obtained from a MRI compatible Luxtron optical fiber thermometer. The probe was placed in the center of the water-bath, and in the periphery of the polyacrylamide center gel, respectively.

\section{MR Data analysis}

Signal noise ratio (SNR) for each sequence was calculated as the mean of the ROI magnitude signal divided by 1.53 times the variance of a similar sized ROI without signal. ROI size was about 1500 voxels.

$\mathrm{T}_{1}$-weighted images before and after heating experiment (figure 3 ) were compared by subtracting the mean of ROIs placed in the centre of each sample. Hence enhancement (\%) was calculated by dividing the signal change by the ROI signal before heating. 
All relaxation times $T_{1}, T_{2}$ or $T_{2}{ }^{*}$ were determined using a Marquardt-Levenberg fit of the function

(1), (2) and (3) respectively, to the corresponding relaxation time measurement data.

1. $M_{z}(T I)=\left|M_{z 0}\left(1-2 e^{\frac{-T I}{T_{1}}}\right)\right|$, with $\mathrm{M}_{\mathrm{z} 0}$ the magnitude for $\mathrm{TI}=0$

2. $M_{x y}(T E)=M_{x y 0} \cdot e^{\frac{-T E}{T_{2}}}$

3. $M_{x y}(T E)=M_{x y 0} \cdot e^{\frac{-T E}{T_{2}^{i}}}$

$\Delta \mathrm{B} 0$ was not taken in consideration for $\mathrm{T}_{2} *$ fitting. To minimize the constant magnetic field variation $(\Delta \mathrm{B} 0)$ effect on $\mathrm{T}_{2} *$ maps the most homogenous map was manually chosen, based on the minimum fit residual.

After having computed relaxation time maps $\mathrm{T}_{1}, \mathrm{~T}_{2}$ and $\mathrm{T}_{2}{ }^{*}$, ROIs were set manually encompassing each voxels corresponding to a single particle solution, excluding the peripheral voxels. Then means and standard deviations of $1 / \mathrm{T}_{1}, 1 / \mathrm{T}_{2}$ and $1 / \mathrm{T}_{2}{ }^{*}$ were calculated. Finally, the particles relaxivities were obtained from error weighted linear regression of $1 / T_{1}, 1 / T_{2}$ and $1 / T_{2}{ }^{*}$ as a function of the particle concentrations. For the before / after heating experiment, measures were done at $18^{\circ} \mathrm{C}$.

All Experiments were run in triplicate and acquired data were expressed as mean \pm SD. Statistical significance was determined using Student's t-test. Differences were considered statistically significant if $\mathrm{P}<0.05$ All calculations were done using custom scripts written in IDL, (Exelis, Boulder, Colorado).

\section{Transmission electron microscopy}

Cryo-TEM is a powerful technique for the visualization of liposomes in the size range from 5 to about 500nm. The magnetoliposome solution was diluted in buffer to reach a phospholipid 
concentration of about $1 \mathrm{mM}$. A single drop $(2 \mu \mathrm{L})$ was deposited on a copper grid covered with a perforated polymer film and a thin carbon layer on both sides. Then most of the liquid was removed with filter paper, leaving a thin film suspended by the grid. The samples were shock-frozen by dipping into liquid ethane and cooled to $90 \mathrm{~K}$ by liquid nitrogen. The samples were transferred to the microscope (JEOL $2100 \mathrm{HC}, 200 \mathrm{kV}$ ) and then examined at approximately $100 \mathrm{~K}$.

\section{ACKNOWLEDGEMENTS}

We thank, Dr. M. Ries for scientific advises, Dr. S. Roujol and Dr. S. Hey for helpful discussions, Ghislaine Frébourg, IFR 83, Université Pierre et Marie Curie for TEM experiments. This work was supported by the European project SonoDrugs (FP7-NMP-2008-1-213706).

\section{REFERENCES}

(1) Carver JR, Shapiro CL, Ng A, Jacobs L, Schwartz C, Virgo KS, Hagerty KL, Somerfield MR, Vaughn DJ. American Society of Clinical Oncology clinical evidence review on the ongoing care of adult cancer survivors: cardiac and pulmonary late effects. J. Clin. Oncol. 2007; 25

(2) Lake RA, Robinson BWS. Immunotherapy and chemotherapy [mdash] a practical partnership. Nat Rev Cancer 2005; 5

(3) Alaouie AM, Sofou S. Liposomes with Triggered Content Release for Cancer Therapy. Journal of Biomedical Nanotechnology 2008; 4

(4) Allen TM, Cullis PR. Drug Delivery Systems: Entering the Mainstream. Science 2004; 303

(5) Ganta S, Devalapally H, Shahiwala A, Amiji M. A review of stimuli-responsive nanocarriers for drug and gene delivery. J Control Release 2008; 126

(6) Fang J, Nakamura H, Maeda $\mathrm{H}$. The EPR effect: Unique features of tumor blood vessels for drug delivery, factors involved, and limitations and augmentation of the effect. Adv. Drug Deliv. Rev. 2011; 63

(7) Bangham AD, Standish MM, Watkins JC. Diffusion of univalent ions across the lamellae of swollen phospholipids. J. Mol. Biol. 1965; 13

(8) Bangham AD. Surrogate cells or Trojan horses. The discovery of liposomes. Bioessays 1995; 17

(9) Andresen TL, Jensen SS, Jørgensen K. Advanced strategies in liposomal cancer therapy: Problems and prospects of active and tumor specific drug release. Progress in Lipid Research 2005; 44

(10) Davis ME, Chen Z (Georgia), Shin DM. Nanoparticle therapeutics: an emerging treatment modality for cancer. Nature Reviews Drug Discovery 2008; 7 
(11) Batist G, Ramakrishnan G, Rao CS, Chandrasekharan A, Gutheil J, Guthrie T, Shah P, Khojasteh A, Nair MK, Hoelzer K, Tkaczuk K, Park YC, Lee LW. Reduced Cardiotoxicity and Preserved Antitumor Efficacy of Liposome-Encapsulated Doxorubicin and Cyclophosphamide Compared With Conventional Doxorubicin and Cyclophosphamide in a Randomized, Multicenter Trial of Metastatic Breast Cancer. JCO 2001; 19

(12) Yatvin M, Weinstein J, Dennis W, Blumenthal R. Design of liposomes for enhanced local release of drugs by hyperthermia. Science 1978; 202

(13) Jolesz FA. MRI-Guided Focused Ultrasound Surgery. Annual Review of Medicine 2009; 60

(14) Staruch R, Chopra R, Hynynen K. Localised drug release using MRI-controlled focused ultrasound hyperthermia. Int J Hyperthermia 2011; 27

(15) Ranjan A, Jacobs GC, Woods DL, Negussie AH, Partanen A, Yarmolenko PS, Gacchina CE, Sharma KV, Frenkel V, Wood BJ, Dreher MR. Image-guided drug delivery with magnetic resonance guided high intensity focused ultrasound and temperature sensitive liposomes in a rabbit $\mathrm{V} \times 2$ tumor model. Journal of Controlled Release Date Missing !!; Volume?(0)

(16) Deckers R, Moonen CTW. Ultrasound triggered, image guided, local drug delivery. Journal of Controlled Release 2010; 148

(17) Bos C, Lepetit-Coiffé M, Quesson B, Moonen CTW. Simultaneous monitoring of temperature and T1: Methods and preliminary results of application to drug delivery using thermosensitive liposomes. Magnetic Resonance in Medicine 2005; 54

(18) de Smet M, Langereis S, den Bosch S van, Grüll H. Temperature-sensitive liposomes for doxorubicin delivery under MRI guidance. Journal of Controlled Release 2010; 143

(19) Smirnov P, Gazeau F, Beloeil J -C, Doan BT, Wilhelm C, Gillet B. Single-cell detection by gradient echo 9.4 T MRI: a parametric study. Contrast Media \& Molecular Imaging 2006; 1

(20) Billotey C, Wilhelm C, Devaud M, Bacri JC, Bittoun J, Gazeau F. Cell internalization of anionic maghemite nanoparticles: quantitative effect on magnetic resonance imaging. Magn Reson Med 2003; 49

(21) Smirnov P. Cellular magnetic resonance imaging using superparamagnetic anionic iron oxide nanoparticles: applications to in vivo trafficking of lymphocytes and cell-based anticancer therapy. Met. Mol. Bio. 2009; 512

(22) Bulte JW, De Cuyper M. Magnetoliposomes as contrast agents. Meth. Enzymol. 2003; 373

(23) Bulte JWM, Cuyper M de, Despres D, Frank JA. Preparation, relaxometry, and biokinetics of PEGylated magnetoliposomes as MR contrast agent. Journal of Magnetism and Magnetic Materials 1999; 194

(24) Lee D. Highly effective T2 MR contrast agent based on heparinized superparamagnetic iron oxide nanoparticles. Macromolecular Research 2011; 19

(25) de Haan HW. Mechanisms of proton spin dephasing in a system of magnetic particles. Magnetic Resonance in Medicine 2011; 66

(26) Demas V, Lowery TJ. Magnetic resonance for in vitro medical diagnostics: superparamagnetic nanoparticle-based magnetic relaxation switches. New Journal of Physics 2011; 13

(27) Brooks RA, Moiny F, Gillis P. On T2-shortening by weakly magnetized particles: The chemical exchange modelt. Magnetic Resonance in Medicine 2001; 45

(28) Brooks RA. T2-shortening by strongly magnetized spheres: A chemical exchange modelt. Magnetic Resonance in Medicine 2002; 47

(29) de Haan HW, Paquet C. Enhancement and degradation of the $\mathrm{R} 2 *$ relaxation rate resulting from the encapsulation of magnetic particles with hydrophilic coatings. Magnetic Resonance in Medicine 2011; 66 
(30) Lasic D. Liposomes: from physics to applications. Elsevier: Amsterdam, New York, 1993

(31) Bacri J-C, Perzynski R, Salin D, Cabuil V, Massart R. Ionic ferrofluids: A crossing of chemistry and physics. J. Magn. Magn. Mat. 1990; 85

(32) Martina M-S, Fortin J-P, Ménager C, Clément O, Barratt G, Grabielle-Madelmont C, Gazeau F, Cabuil V, Lesieur S. Generation of superparamagnetic liposomes revealed as highly efficient MRI contrast agents for in vivo imaging. J. Am. Chem. Soc. $2005 ; 127$ 


\section{FIGURE CAPTIONS}

Figure 1. Cryo-TEM images of thermosensitive magnetoliposomes (TSM) before (a) and after (b) heating to $45^{\circ} \mathrm{C}$ for $10 \mathrm{~min}$ (scale bar: $0.1 \mu \mathrm{m}$ ). Before heating, the intact spherical liposome membranes can be observed, (solid arrows) encapsulating iron oxide nanoparticles (dashed arrow). USPIO are contained in the liposomes to form aggregates (curly bracket). After heating, we can observe scattered free USPIO particles (open arrow) and empty, dented phospholipids bilayers, (dashed open arrow) respectively. Size distribution of TSM determined by Dynamic Light Scattering (distribution is represented in intensity of scattered light) (c).

Figure 2. Relaxation rates $R_{1}$ (a), $R_{2}$ (b), $R_{2} *(c)$ vs. iron concentration for determination of relaxivities $r_{1}, r_{2}$ and $r_{2}{ }^{*}$ of TSM. A linear fit allows determining the relaxivities $r_{1}, r_{2}, r_{2}{ }^{*}$. Relaxivities $r_{1}(d), r_{2}(d)$ and $r_{2} *(f)$ of thermosensitive magnetoliposomes (TSM) and free USPIO, respectively, before (solid bars) and after (hatched bars) heating to $45^{\circ} \mathrm{C}$ for 10 minutes.

Figure 3. $\mathrm{T} 1$-weighted gradient echo images $(\mathrm{TE}=10 \mathrm{~ms})$ before and after water bath heating to $45^{\circ} \mathrm{C}$, for thermosensitive liposomes, non-thermosensitive liposomes and free iron oxide particles. A clear MRI signal enhancement of $83 \pm 4 \%$ is shown for TSM. Dotted white circle shows typical ROI used for enhancement calculation.

Figure 4. MR-signal enhancement observed during water bath heating as function of time (a), for TSM (black squares), and free USPIO (grey squares), for the samples with $20 \mu \mathrm{g} / \mathrm{ml}$ iron concentration. The thin line shows the theoretical magnitude enhancement based on observed $\mathrm{T}_{2}{ }^{*}$ evolution, assuming a fixed $\mathrm{T} 1$. The sigmoidal variations are limited by two vertical lines at $42^{\circ} \mathrm{C}$ and $44.5^{\circ} \mathrm{C}$, a transition which took place in 7:30 minutes (double open arrow). Evolution of T1-weighted MR signal vs. PRFS temperature during the experiment (b), for the heating (solid lines) and subsequent cooling phases (dashed lines). Solid and dashed arrows (corresponding to the heating and cooling period respectively) indicate the evolution of time. As opposed to the MR-signal 
enhancement evolution from USPIO (grey line), MR-signal from TSM (black line) present a hysteresis, indicating that an irreversible change took place.

Figure 5. Relaxivity $r_{2}{ }^{*}$ of TSM (black square) and Free USPIO (grey square) produced during a water bath heating experiment as a function of time (a). Relaxivity $r_{2} *$ evolution during heating (solid lines) and cooling (dashed lines) of the sample as a function of temperature for TSM (black lines) and free USPIO (grey lines) (b). For $\mathrm{r}_{2}{ }^{*}$, as well, the TSM relaxivity shows a hysteresis effect, indicating an irreversible change after the heating cycle.

Figure 6. Magnitude images ( $\mathrm{a}, \mathrm{c})$ and $\mathrm{T}_{2} *$ maps $(\mathrm{b}, \mathrm{d})$ obtained during a HIFU heating experiment, before $(a, b)$ and 15 minutes after HIFU application (c, d), respectively. The first location (white solid line) received 4 heating cycles, the second location (white dashed line) received a single heating cycle. 

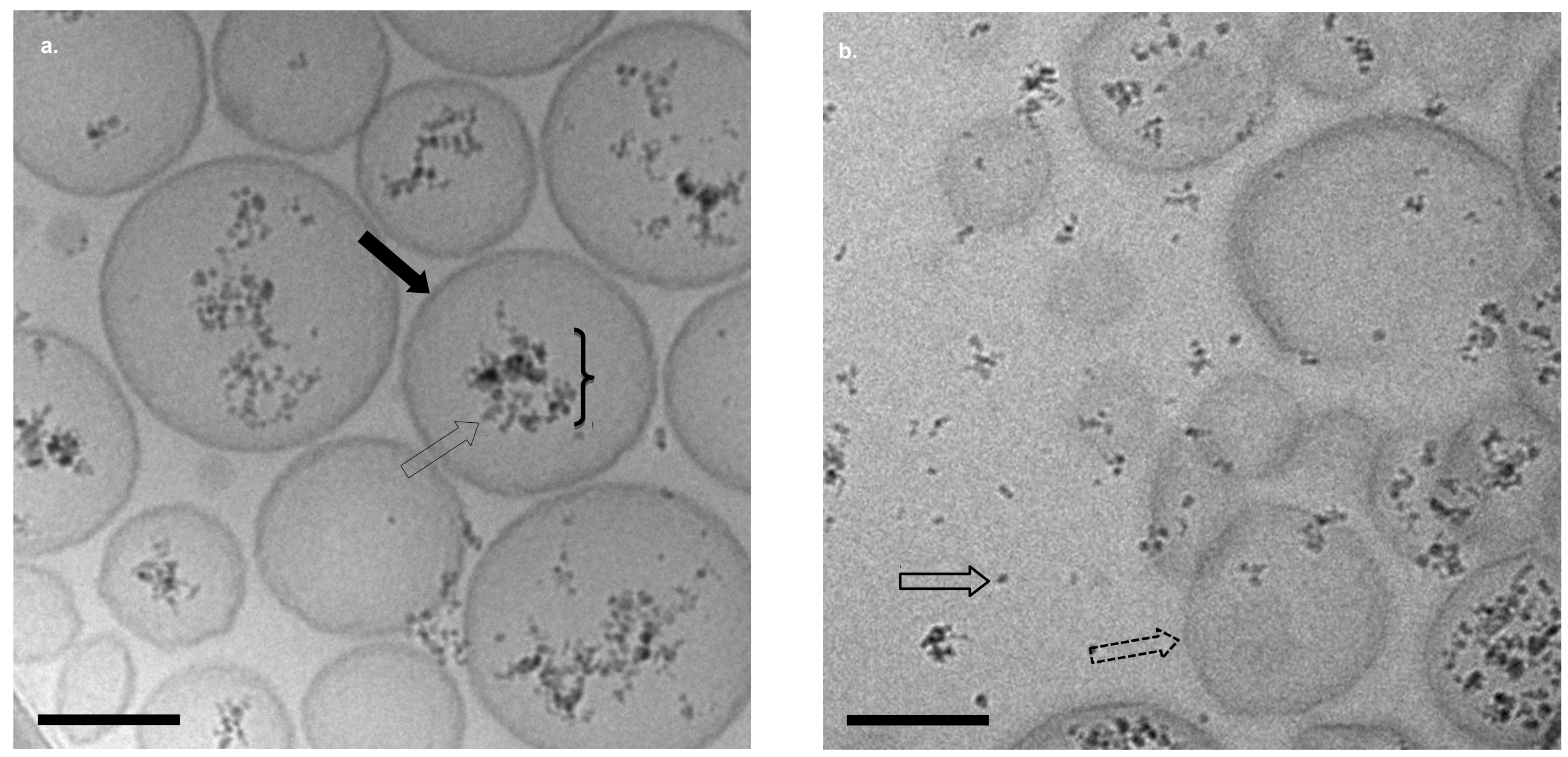

c.

Size distributionintensity

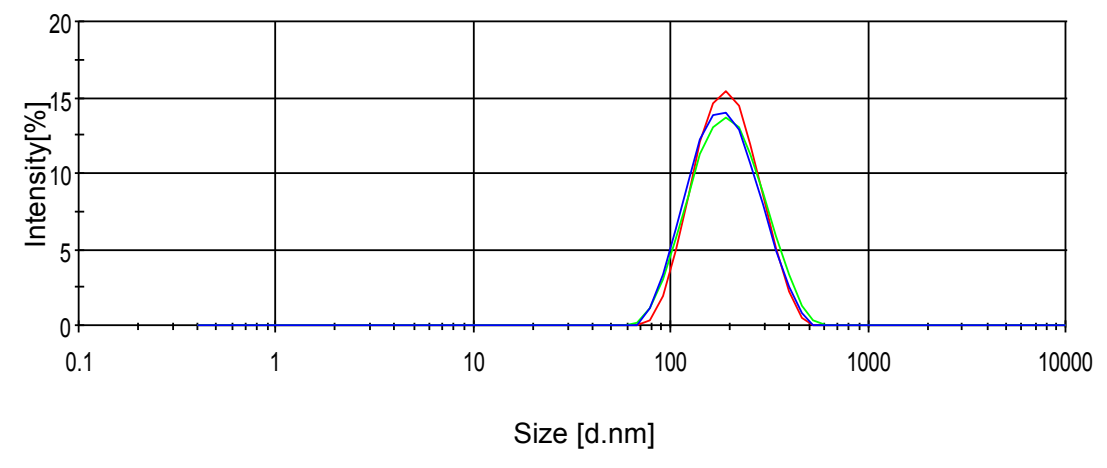


a.

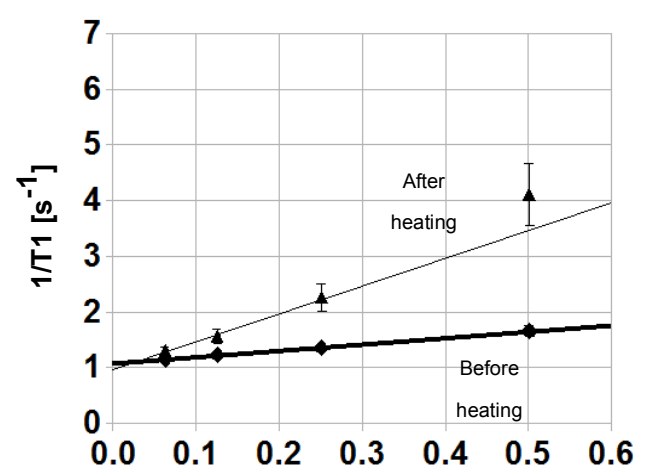

d.

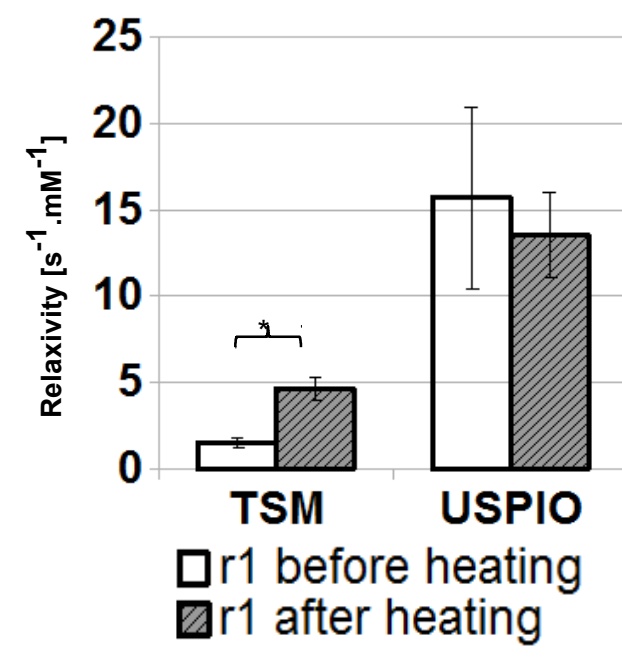

b.

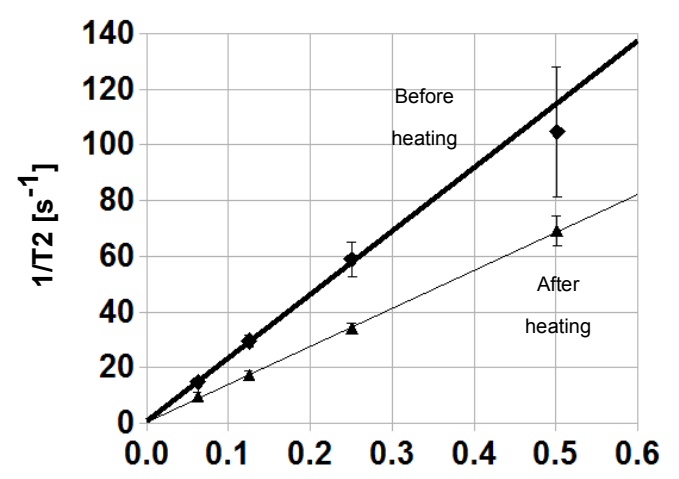

Concentration [mM]

e.

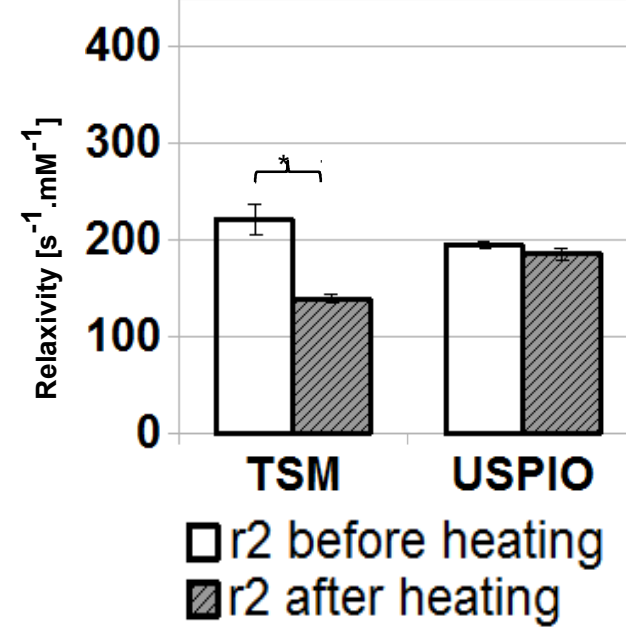

c.

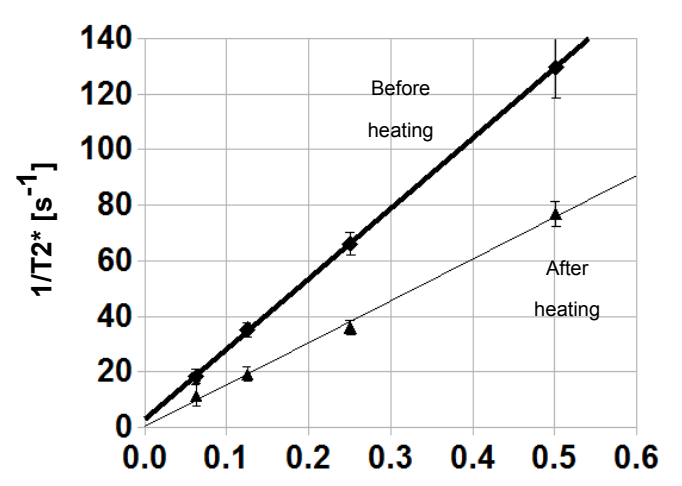

f.

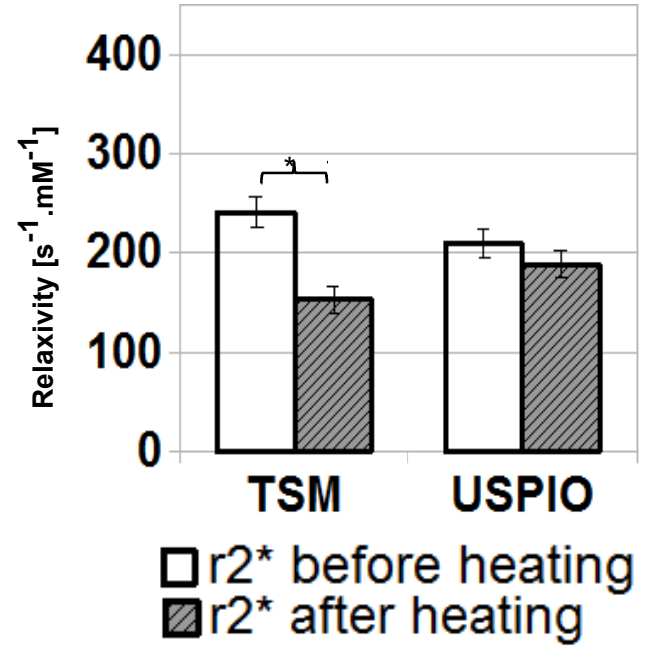




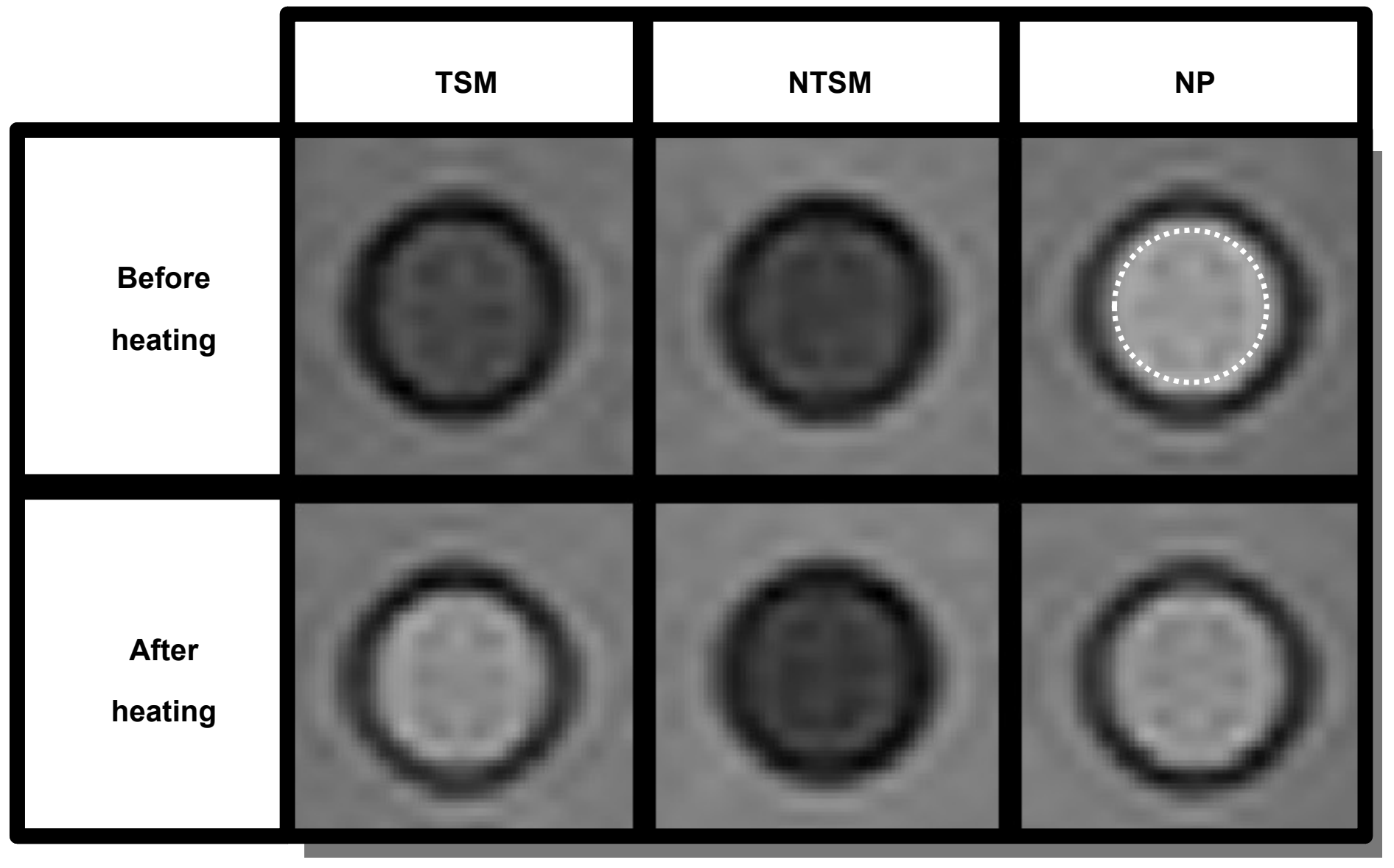




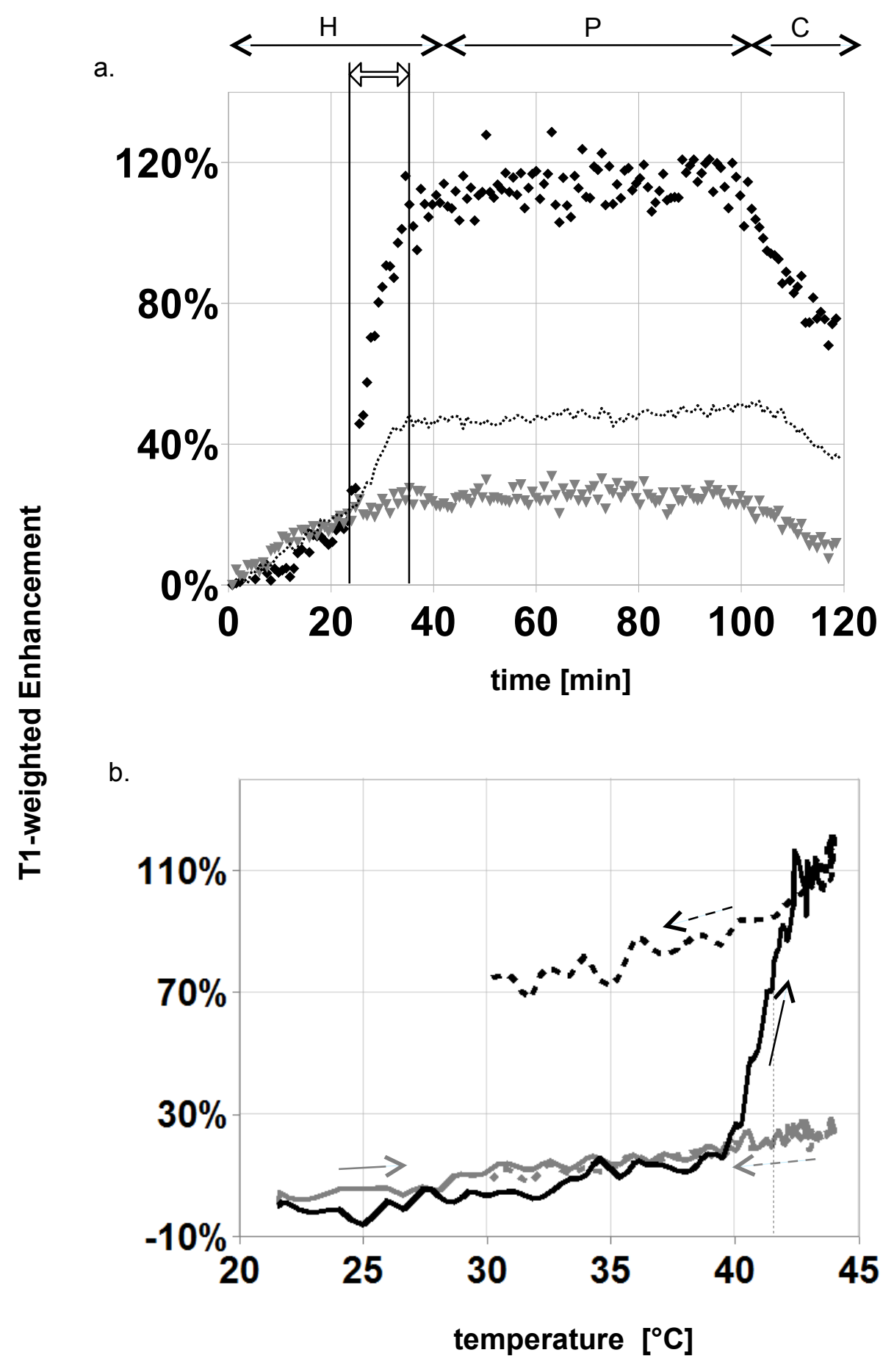




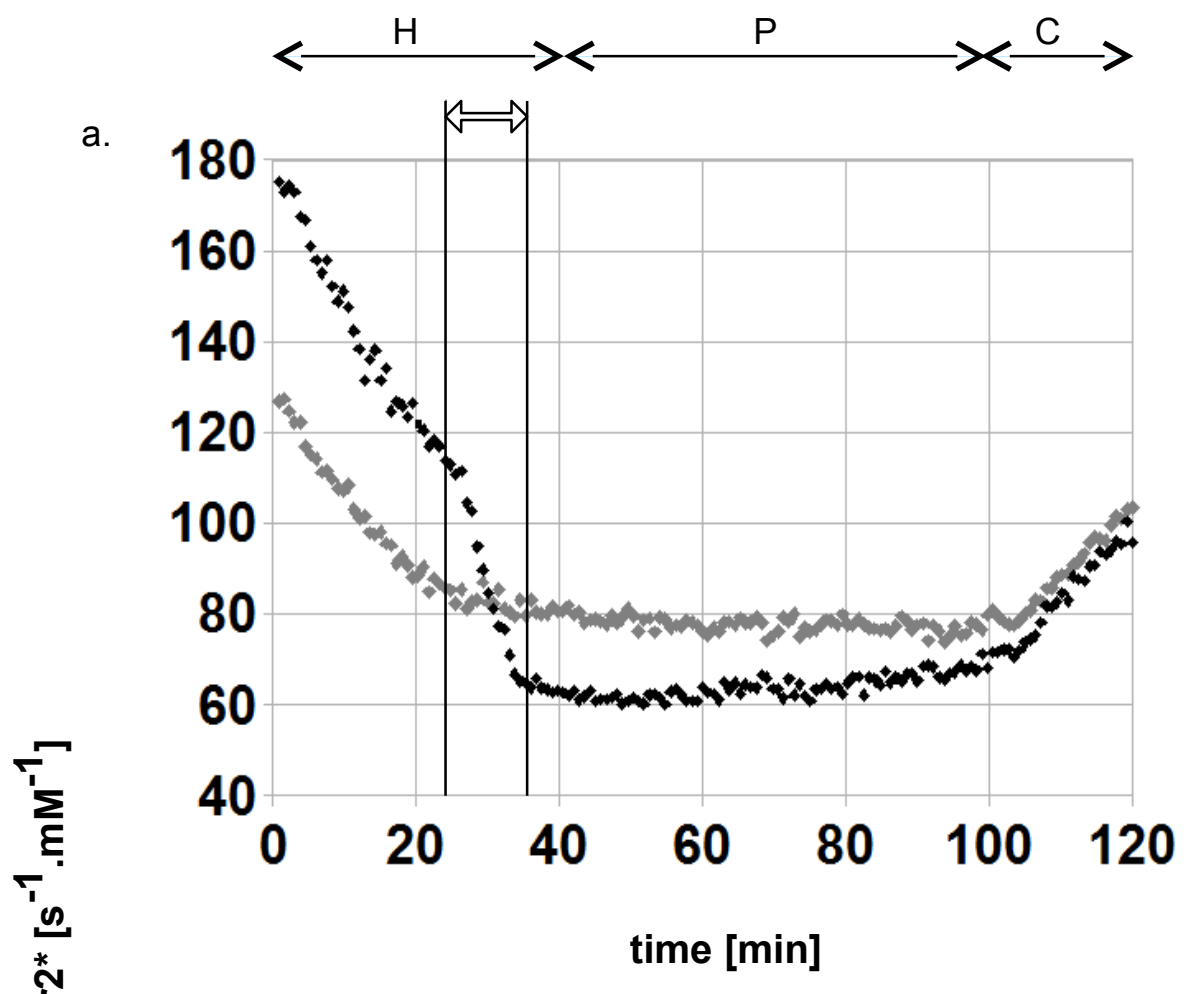

b.

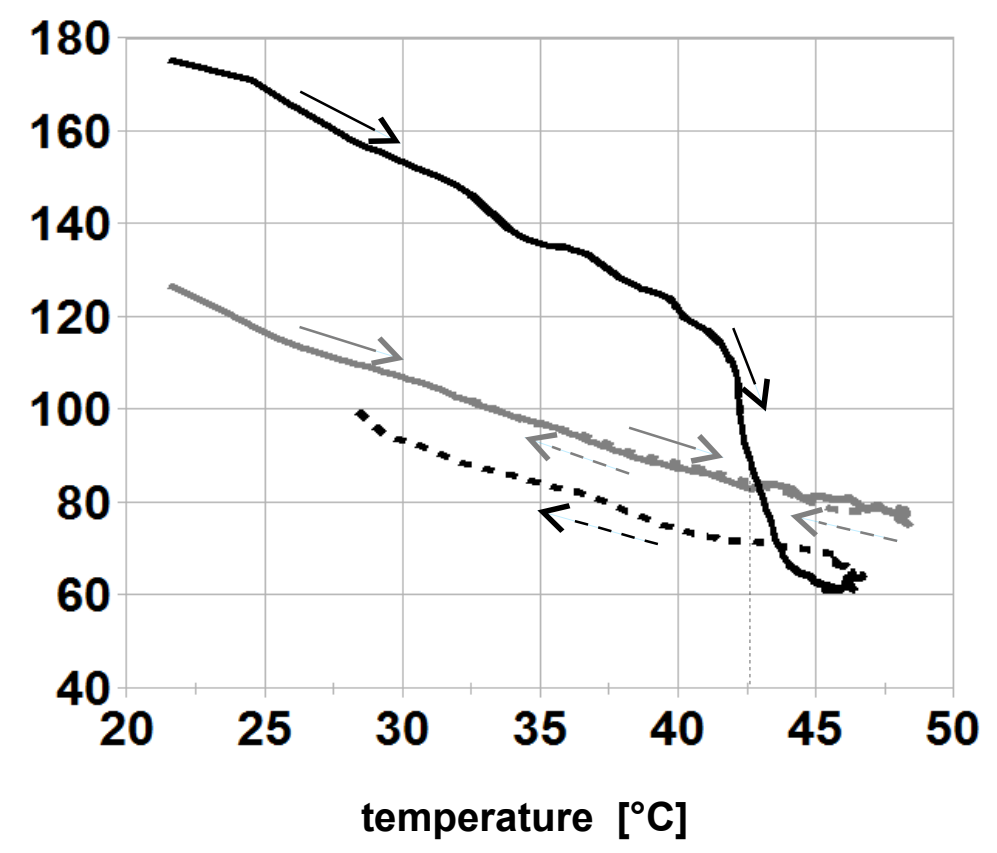

$\mathrm{H}$ : heating

P: plateau

C: cooling 
a.

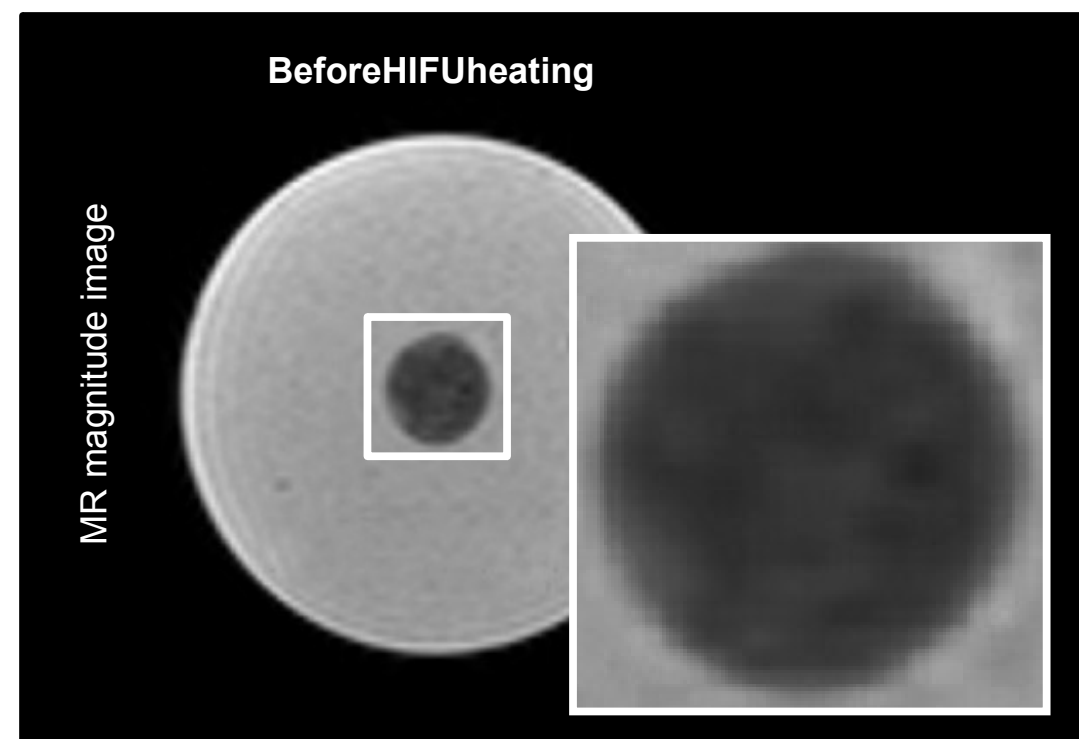

b.

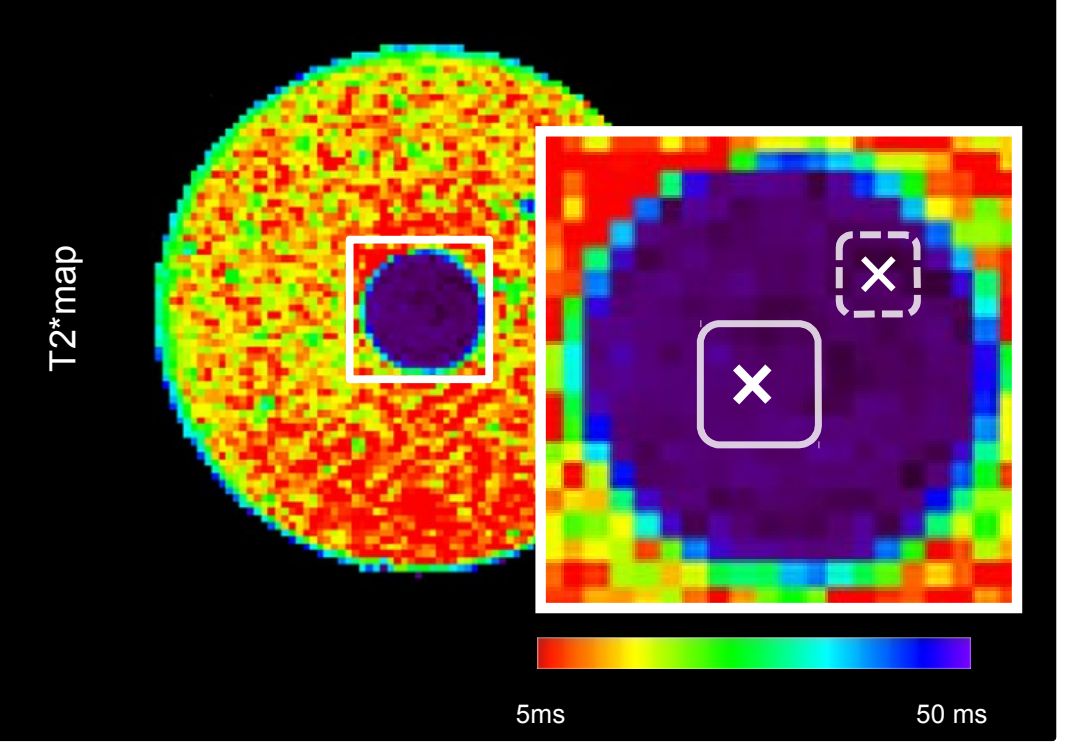

c.

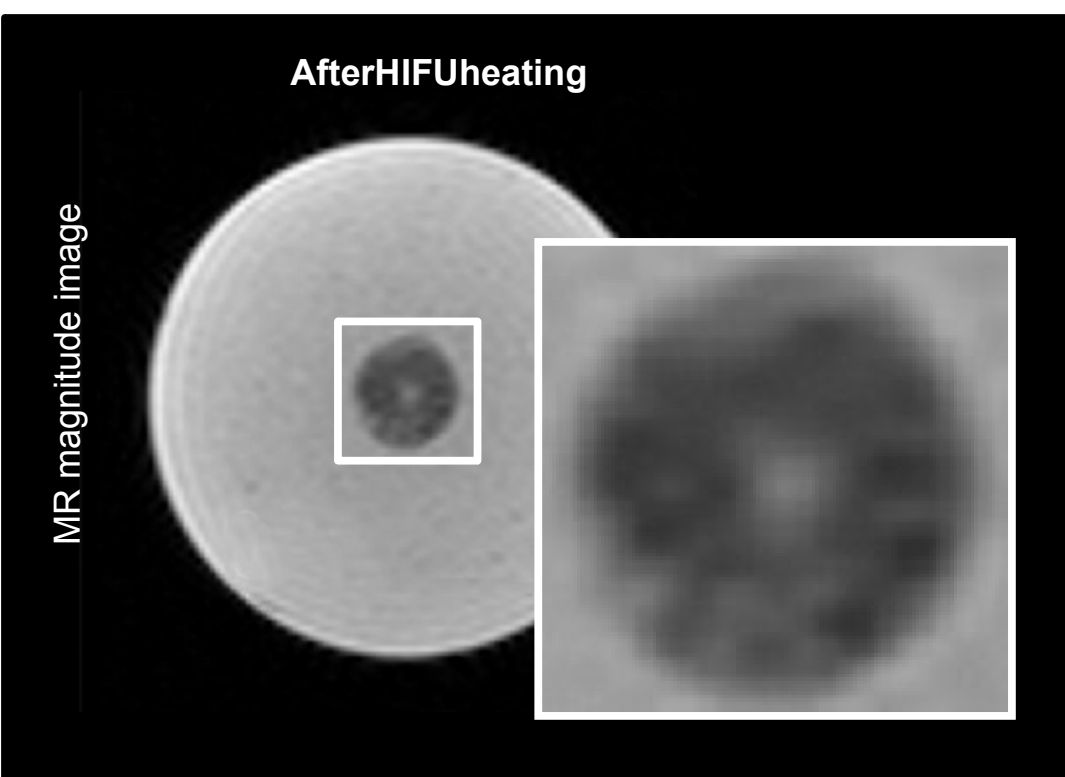

d.

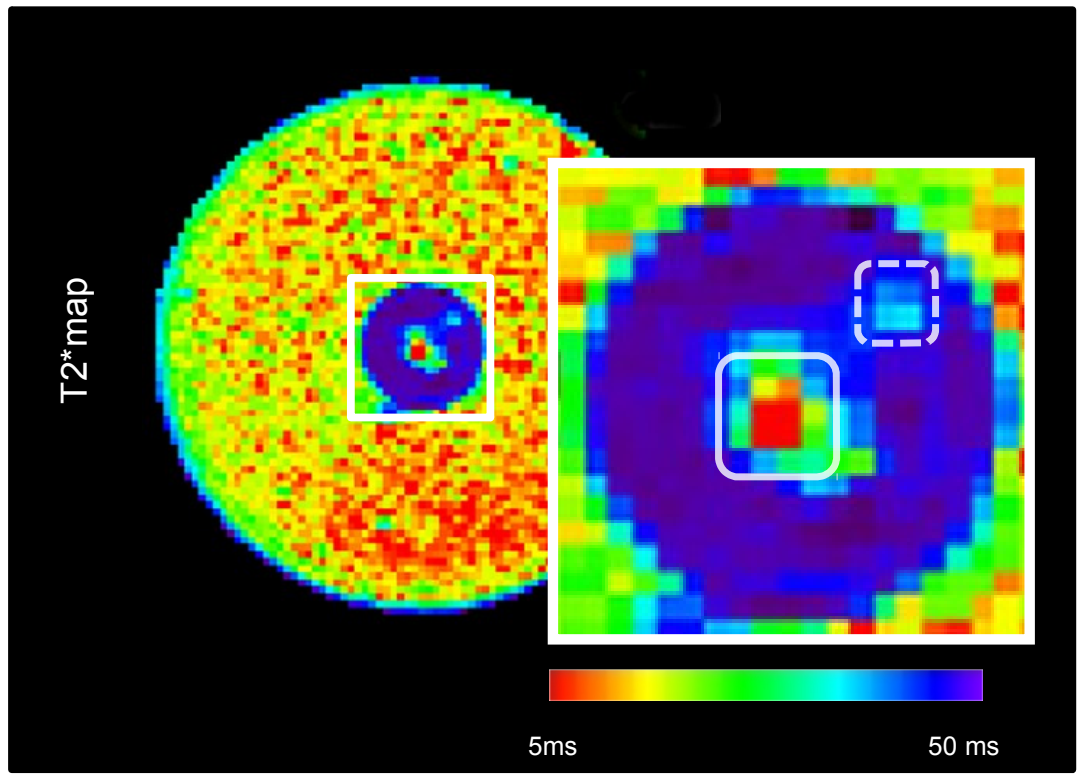

